\title{
FASA - Fire Airborne Spectral Analysis of natural disasters
}

\author{
Erwin Lindermeir $\left({ }^{1}\right)$, Hermann Kick $\left({ }^{1}\right)$, Volker Tank $\left({ }^{1}\right)$, Peter Haschberger $\left({ }^{1}\right)$, \\ Maria Fabrizia Buongiorno $\left({ }^{2}\right)$, Giuseppe Distefano $\left({ }^{2}\right)$, Stefania Amici $\left({ }^{2}\right)$, Dieter Oertel $\left({ }^{3}\right)$, \\ Friedrich Schrandt $\left(^{3}\right)$, Wolfgang Skrbek $\left({ }^{3}\right)$ and Holger Venus $\left({ }^{3}\right)$ \\ (') DLR - German Aerospace Center, Remote Sensing Technology Institute, Wessling, Germany \\ (') Istituto Nazionale di Geofisica e Vulcanologia, Roma, Italy \\ $\left(^{3}\right)$ DLR - German Aerospace Center, Institute of Space Sensor Technology \\ and Planetary Exploration, Berlin, Germany
}

\begin{abstract}
At present the authors are developing the system FASA, an airborne combination of a Fourier Transform Spectrometer and an imaging system. The aim is to provide a system that is usable to investigate and monitor emissions from natural disasters such as wild fires and from volcanoes. Besides temperatures and (burned) areas FASA will also provide concentration profiles of the gaseous combustion products. These data are needed to improve the knowledge of the effects of such emissions on the global ecosystem. The paper presents a description of the instrumentation, the data evaluation procedure and shows first results of retrieval calculations based on simulated spectra.
\end{abstract}

Key words remote sensing - wild fires - volcano emissions - Fourier spectrometry - data-fusion

\section{Introduction}

Fires change ecosystems in all vegetation zones. The concern of the public, politicians and scientists about climate change and environmental hazards has stressed the need for a better understanding of the dynamics of biomass burning and volcanic activities at all scales from local to global, in order to estimate the role of fire as a contributor to that change. Recent publications indicate that biomass burning may be a major source of greenhouse gases (Page et al., 2002).

Mailing address: Dr. Erwin Lindermeir, DLR - German Aerospace Center, Remote Sensing Technology Institute, P.O. Box 1116 D-82230 Wessling, Germany; e-mail: Erwin.Lindermeir@dlr.de
However, large uncertainties in the quantification of these emissions exist.

Airborne remote sensing is the predestined method to investigate natural disasters. It permits riskless measurements and coverage of large and otherwise often difficult to access areas. However, by now mainly space borne sensors that were not developed for HTE investigations were used. They often suffer from detector saturation and low spatial resolution. Usually they permit only the detection of larger fires and provide only surface temperatures and the size of the burnt areas; but no direct information with regard to the emitted gases.

The Italian Instituto Nazionale di Geofisica e Vulcanologia (INGV) and the German Aerospace Center (DLR) cooperate in developing new airborne instrumentation specifically for HTE analyses. This system is called FASA (Fire Airborne Spectral Analyser). The objective is to determine not only temperatures and areas; beyond these parameters the quantification of the released gases and aerosols is to be achieved. 
The task requires the use of a sensor system that provides high spatial and high spectral resolution simultaneously. The following sections first present the choices made for the instrumentation and secondly the principles of the data fusion algorithms as well as a first simulation results.

\section{Instrumentation}

\subsection{Concept}

At first glance one might be tempted to consider the use of an imaging spectrometer, which provides spatial and spectral resolution simultaneously. However, so as to retrieve concentration profiles it is necessary to resolve the single emission lines of the gases in the spectra. Their line widths are in the order of $0.1 \mathrm{~cm}^{-1}$. In addition, burning areas exhibit a quite high degree of spatial variation which leads to the demand for an accordingly high spatial resolution $(\sim 1 \mathrm{~m})$ of the sensor. As the retrieval algorithms which are used to derive concentrations and temperatures from the spectra need a certain SNR level to produce high quality results, the use of an imaging spectrometer for this application will suffer from a distribution problem: the received energy can either be used to obtain low noise spectra at high spectral resolution, or low noise images at high spatial resolution.

Therefore it was decided to use two specialized instruments for the two tasks: a three channel imaging system and a Fourier transform spectrometer. The next two sections describe these instruments in more detail.

\subsection{MIROR Fourier transform spectrometer}

The airborne Michelson Interferometer with Rotating Retro-reflector (MIROR) was originally designed and built by DLR to investigate emissions of aircraft engines in flight (Haschberger and Lindermeir, 1996). It is therefore very well suited to record spectra of hot combustion products. The spectra provided by MIROR have already been successfully used to determine temperature and concentration pro- files in the plumes of aircraft jet engines (Lindermeir et al., 2001).

Operating a Michelson interferometer in a harsh environment such as on board an aircraft is a challenge for the optical set-up of the instrument. The quality of the interferometer's detector signal strongly depends on the precise position of the optical components. Permissible tolerances are below the wavelengths of the incident radiation, i.e. less than a micrometer for infrared applications. From the mechanical point of view the necessary movement of one of the optical components further complicates the problem. Consequently, Michelson interferometers are routinely used mainly under laboratory conditions.

With the MIROR design a very rugged optical set-up was realized which additionally overcomes the stop-and-go movement of other solutions. The most advanced of the MIROR setups is depicted in fig. 1 from which the heritage from the classical Michelson set-up is still obvious. There are the beamsplitter, the two mirrors, which are labelled 'mirror with hole', and the detector with appropriate optics. In addition to these classical components two mirrors direct the two beams which the beamsplitter generates into a retro-reflector. This is an optical element consisting of three plane mirrors which are perpendicular to each other, i.e. they form the corner of a cube. The retro-reflector property, which is exploited by the MIROR principle, is that beams are reflected in the same direction in which they entered the reflector. The reflector only introduces a shift to the side. This effect is independent of the angle of incidence. The two beams from the beamsplitter are therefore reflected back to the mirrors with the holes from where they are sent back to the beamsplitter. Here they recombine and the interference pattern finally reaches the detector.

During a measurement the reflector is spinning at constant angular velocity around the axis. As indicated in fig. 1 the reflector's centre is located at a distance $L$ from the axis of rotation. As shown in (Haschberger, 1992) this causes a continuous change of pathlengths for the two beams and thus the interferogram is generated.

As mentioned above the ruggedness of the DLR MIROR spectrometer was already shown. 


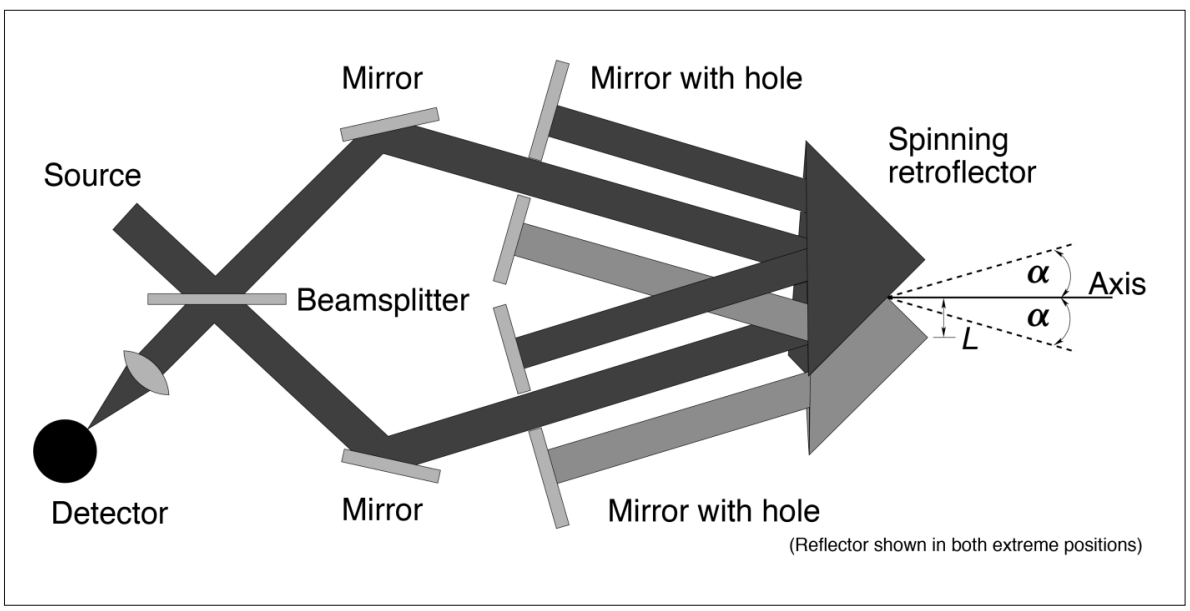

Fig. 1. Optical set-up of the MIROR Fourier transform spectrometer.

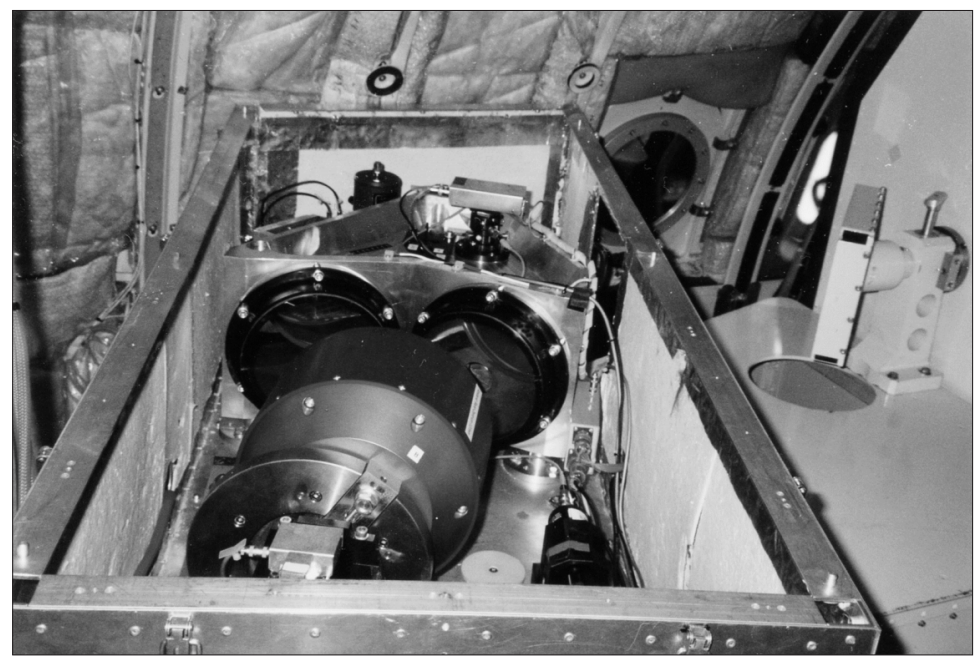

Fig. 2. Optical set-up of the MIROR spectrometer installed in one of DLR's research aircrafts.

It provided high quality spectra not only under flight conditions but also during jet engine runs in test rigs on the ground. These latter tests were extreme, as the spectrometer was not only subject to vibrations but also to high levels of acoustic noise. Figure 2 shows a photograph of the optical set-up installed in a DLR research aircraft. The technical data of MIROR are summarized in table I.

For the FASA experiment MIROR will be used in a NADIR configuration. Table II provides the footprint diameter on the ground and 
Table I. Technical data of the MIROR Fourier transform spectrometer.

\begin{tabular}{cc}
\hline \hline Spectral range & $600-3000 \mathrm{~cm}^{-1}$, \\
& $(3.3-16.6 \mu \mathrm{m})$ \\
Spectral resolution & $0.2 \mathrm{~cm}^{-1}$ \\
Registration time & $0.22 \mathrm{~s}$ \\
per interferogram & $3 \mathrm{~Hz}$ \\
Measurement rate & $20 \mathrm{mrad}$ \\
Field of View & $6.2 \times 10^{-8} \mathrm{~W} /\left(\mathrm{cm}^{2} \mathrm{sr} \mathrm{cm}^{-1}\right)$ \\
NESR (single scan)
\end{tabular}

Table II. Footprint diameter and smear for various flight altitudes above ground.

\begin{tabular}{ccc}
\hline $\begin{array}{c}\text { Flight altitude } \\
\text { above ground } \\
(\mathrm{m})\end{array}$ & $\begin{array}{c}\text { MIROR footprint } \\
\text { diameter } \\
(\mathrm{m})\end{array}$ & $\begin{array}{c}\text { Footprint smear } \\
\text { for 75 m/s speed } \\
\text { over ground (\%) }\end{array}$ \\
\hline 2000 & 40 & 27.5 \\
3000 & 60 & 14 \\
4000 & 80 & 7 \\
\hline
\end{tabular}

the smear for some flight altitudes. Typically these will be in the range 3000 to $4000 \mathrm{~m}$.

\subsection{ABAS Advanced Bird Airborne Simulator}

ABAS consists of the MIR/TIR Hot Spot Recognition Sensor (HSRS) and VIS/NIR Wide-Angle Airborne Camera (WAAC).

HSRS is a bi-spectral push-broom scanner with spectral bands in the MWIR at $3.9 \mu \mathrm{m}$ and TIR at $8.8 \mu \mathrm{m}$. The sensitive devices are 2 MCT photodiode lines. The lines - with identical layout in the MWIR and TIR - comprise $2 \times$ $\times 512$ elements each in a staggered structure.

These arrays have to be cooled to $100 \mathrm{~K}$ in the MWIR and to $80 \mathrm{~K}$ in the TIR. The maximum achievable at $80 \mathrm{~K}$ TIR photodiode cut-off wavelength of about $10 \mu \mathrm{m}$ and the atmospheric ozone band at $9.6 \mu \mathrm{m}$ make it necessary to use the 8.59.3 $\mu \mathrm{m}$ band for TIR channel of the HSRS instead of the commonly used 10.5-11.7 $\mu \mathrm{m}$ band. The cooling must be conducted in a satellite mission by small Stirling cooling engines. HSRS sensor head components of both spectral channels are based on identical technologies to provide good pixel co-alignment. Both spectral channels have the same optical layout but with different wavelength-adapted lens coatings.

The HSRS sensor data are read out continuously with a clock time which is exactly one half the pixel dwell time. This time controlled «double sampling» and the staggered line array structure allow a geometric resolution enhancement compared to a classic line array.

Radiometric fire measurements require a large dynamic range, as outlined above. To fulfil this requirement, a second scene sampling has to be performed with reduced exposure time (within the same sampling clock time interval!) if hot areas are identified during the real-time processing of the data from the first scene sampling. This second sampling is only performed if the realtime processing of the first sampling set indicates that detector elements are saturated or close to saturation. These supplementary sampled pixels are added to the normal thermal scene measurement data as the so called «Hot Area (HA)» data sub-set. All these sampled data are transmitted to the HSRS data acquisition controller via a special serial interface. In the result, the HSRS has an adaptive radiometric dynamic range which allows the recognition of High Temperature Events (HTE) such as wildfires, volcanic activities, or coal seam fires without sensor saturation.

Table III shows the main technical data of ABAS and table IV lists the swath widths and pixel sizes on the ground for various flight altitudes.

Table III. ABAS technical data.

\begin{tabular}{lll}
\hline \hline Spectral range & VIS/NIR: & $0.4-0.6 \mathrm{~nm}$ \\
& MIR: & $3.4-4.1 \mu \mathrm{m}$ \\
& TIR : & $8.5-9.3 \mu \mathrm{m}$ \\
Field of View & VIS/NIR: & $80^{\circ}$ \\
& MIR/TIR: & $19^{\circ}$ \\
Detector elements & VIS/NIR: & 5184 \\
number (no. of pixels/line) & MIR/TIR: & $2 \times 512$ \\
& & $($ staggered) \\
Instantaneous & VIS/NIR: & $0.27 \mathrm{mrad}$ \\
Field of View & MIR/TIR: & $0.66 \mathrm{mrad}$ \\
\hline
\end{tabular}


Table IV. ABAS-Swath width and pixel sizes for various flight altitudes above ground.

\begin{tabular}{ccccc}
\hline \hline \multirow{2}{*}{ Flight altitude above ground $(\mathrm{m})$} & \multicolumn{2}{c}{ Swath width $(\mathrm{m})$} & \multicolumn{2}{c}{ Linear pixel size $(\mathrm{m})$} \\
& VIS/NIR & MIR/TIR & VIS/NIR & MIR/TIR \\
\hline 2000 & $\sim 3300$ & $\sim 660$ & $\sim 0.7$ & $\sim 1.4$ \\
3000 & $\sim 5000$ & $\sim 1000$ & $\sim 1.0$ & $\sim 2.0$ \\
4000 & $\sim 6600$ & $\sim 1320$ & $\sim 1.4$ & $\sim 2.8$ \\
\hline
\end{tabular}
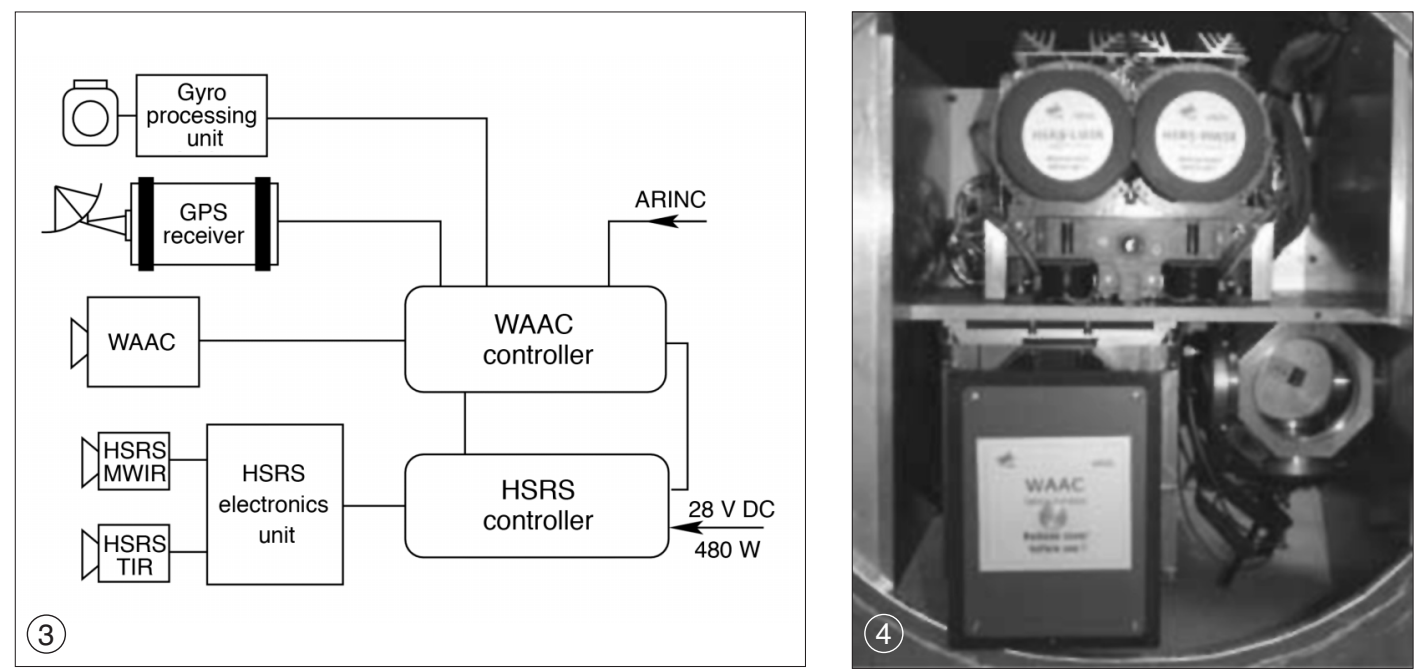

Fig. 3. Block diagram of the BIRD airborne simulator.

Fig. 4. ABAS sensor platform.

Figure 3 depicts a block diagram of the ABAS components. In addition to the cameras there are a GPS receiver and a gyro processing unit which aid in geo-referencing the recorded images. ABAS is also connected to the aircraft's ARINC bus which provides additional (house keeping) data from the aircraft such as temperature and pressure at flight altitude, true air speed and the like. A photo of ABAS is provided with fig. 4.

\subsection{Co-alignment and synchronisation}

As both systems are installed as mechanically independent instruments in the aircraft, it is necessary to align their measurement spots (co-alignment). Furthermore, synchronisation of the recording of spectra and images in time is required.

The latter task is solved by establishing a common time base, i.e. the GPS time of ABAS. In addition, MIROR is provided with an electrical signal that permits counting of the currently recorded image line of ABAS' VIS/NIR channel. This line counter is stored together with the images and the interferograms. Thus the correct assignment of the data from both systems is guaranteed.

Co-alignment is established by also using the VIS/NIR channel: a small video camera equipped with the same optical filter is fixed at the optical block of the spectrometer. Its line of sight is directed in parallel to the spectrometer's line of 
sight. The camera's FoV is about 10 times larger than the spectrometer's FoV. The area observed by the spectrometer in the video camera images is determined before flight in the laboratory.

During flight the video images are recorded simultaneously with the interferograms and the ABAS images. By applying well known techniques (e.g., correlation) the spectrometer FoV is located in the ABAS images.

\section{Data fusion algorithm}

Estimating High Temperature Event (HTE) emission characteristics based on NADIR FTIR measurements is a challenging problem.

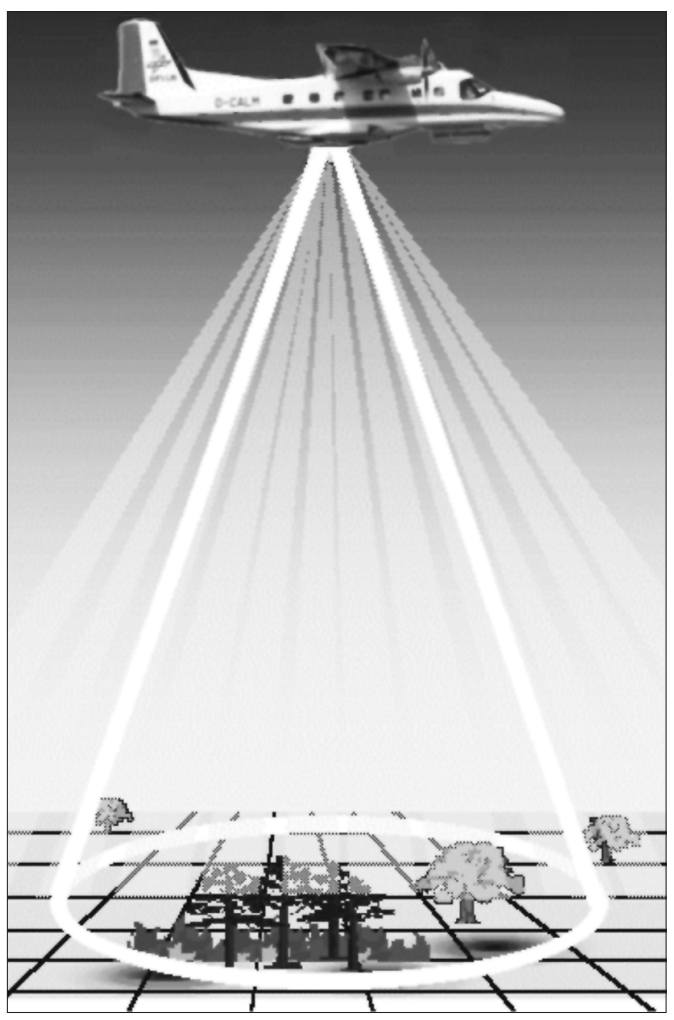

Fig. 5. Footprints of MIROR and ABAS. ABAS determines the distribution of the surface radiance within the MIROR footprint.
First, the retrieval of temperature and concentration profiles from spectroscopic measurements obtained by vertical sounding is well known to be an ill-posed problem, and advanced numerical techniques have to be used to find meaningful solutions. In addition, due to the limited spatial resolution of Fourier transform spectrometers, HTE scenes observed by the NADIR viewing FTS will be horizontally inhomogeneous, i.e., in case of fire observations the observed spectrum will be a superposition of contributions of flaming, smouldering, smoky, and unperturbed areas.

For the solution of ill-posed problems there are several algorithms available to obtain solutions. During the last years a method based on the 'Generalised singular value decomposition' was developed at DLR and will be applied for FASA. A detailed description of this method is available in Schimpf (1999).

The problem caused by scene inhomogeneities can be solved by combining the MIROR spectra with the images provided by the ABAS system. This 'data-fusion' will be based on a sequential approach.

In a first step the data obtained by the imaging system is used to analyse and classify the FTIR footprint area. In this manner the images provide essential information for the retrieval of gas parameters of the HTE, i.e. surface composition (distribution of flaming, smouldering, etc.) and temperature estimates. Based on this knowledge an initial guess for the iterative nonlinear least squares retrieval problem is obtained. This calculation finally yields the temperature and concentration profiles of the HTE.

The proceeding described above is visualized in fig. 5 and fig. 6.

\subsection{Test calculation}

Retrieval tests conducted at DLR have demonstrated the feasibility of the proceeding outlined above. Here the result of one such calculation is briefly described.

It was assumed that the spectrometer's FoV is filled by a flaming, a smouldering, and an undisturbed zone plus smoke. The exact fractions of two cases are shown in fig. 7. The pa- 


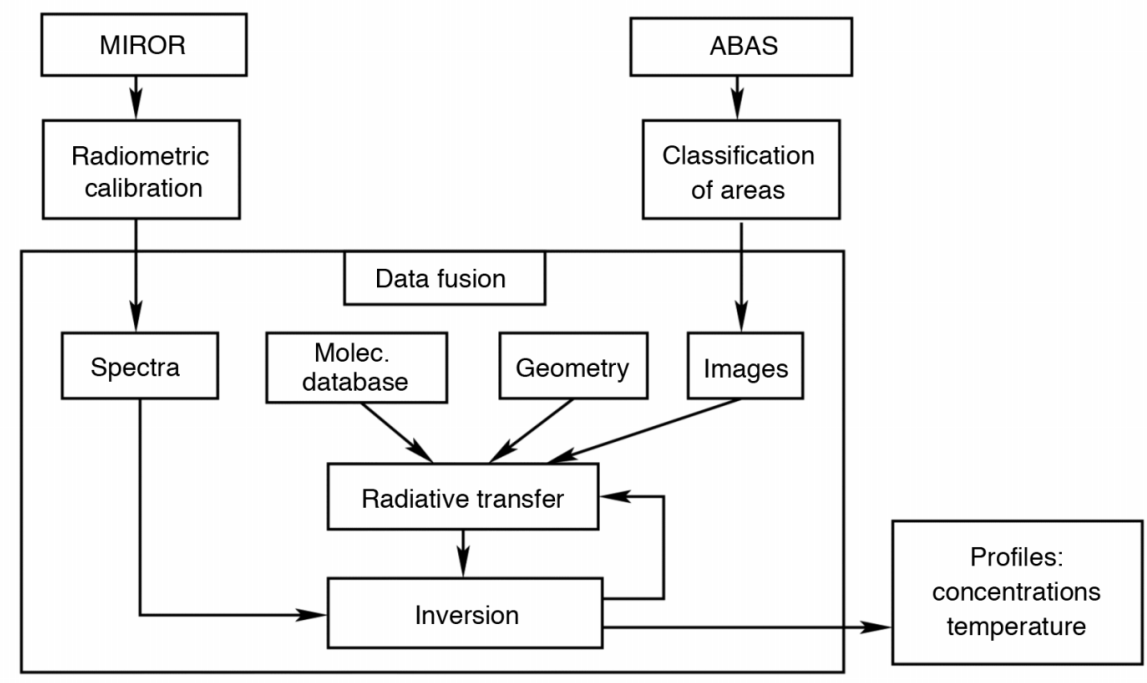

Fig. 6. Schematic visualizing the FASA data fusion algorithm.

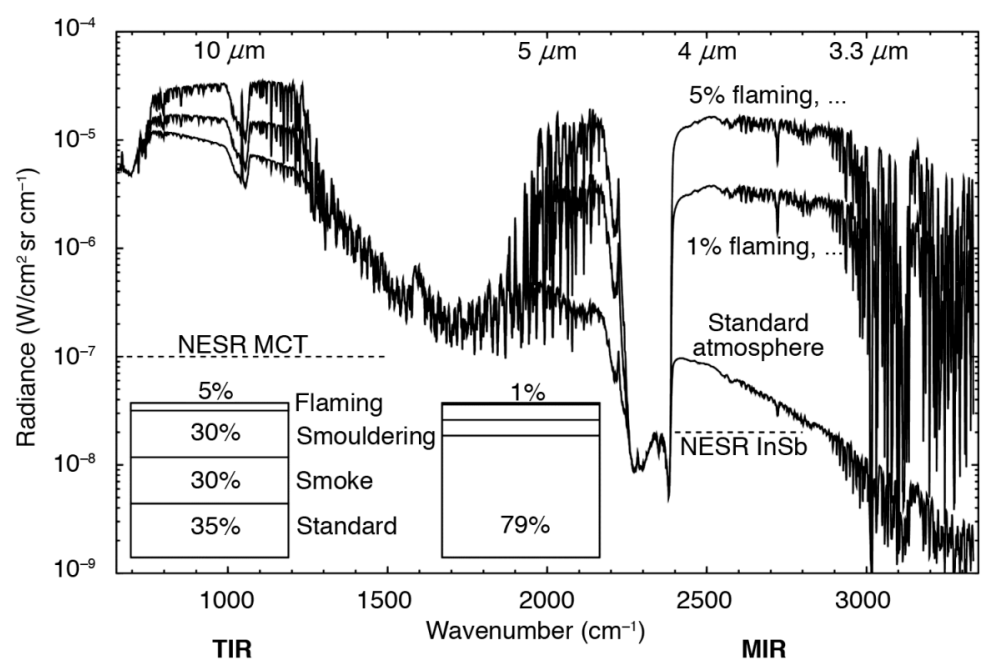

Fig. 7. Radiance spectra calculated for an undisturbed atmosphere and two different compositions of the spectrometer footprint as indicated.

rameters describing the different zones are taken from various papers (Pereira and Setzer, 1996; Stocks et al., 1996; Worden et al., 1997). With regard to gases this investigation considered only $\mathrm{H}_{2} \mathrm{O}, \mathrm{CO}_{2}$, and $\mathrm{CO}$. The spectra in fig.
7 show high levels of contrast in the spectral regions in which these gases have emission lines as well as a generally increased signal level in the window regions which is caused by radiance from the hot surface. 


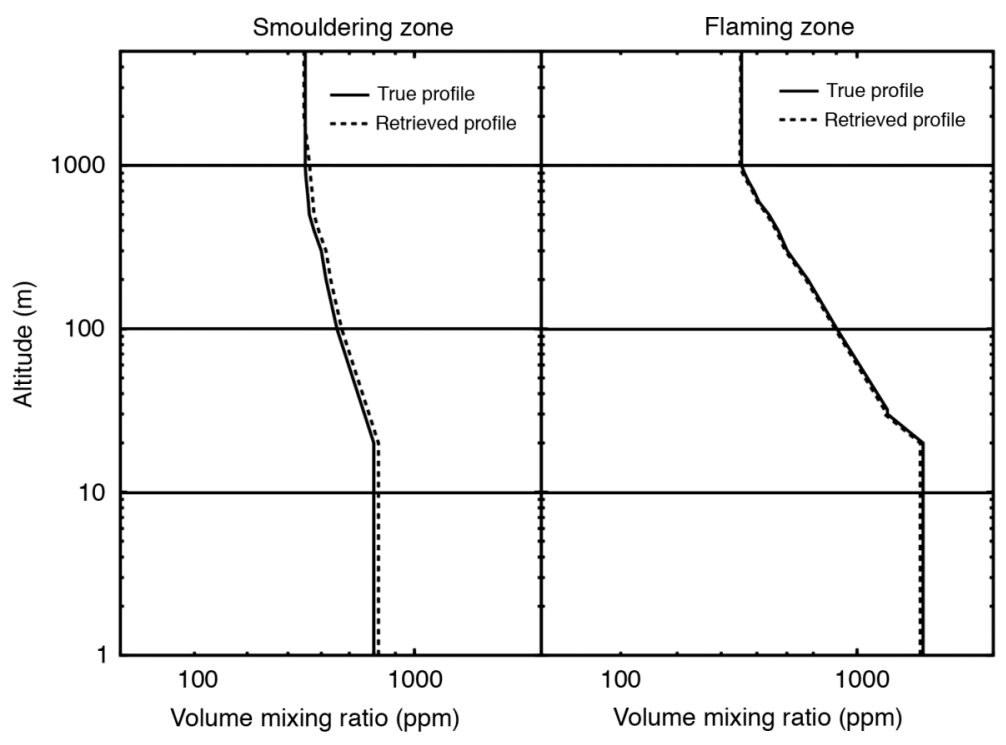

Fig. 8. Retrieval results for the «5\% flaming zone» case: $\mathrm{CO}_{2}$ profiles for the flaming and the smouldering zones.

Figure 8 shows retrieved $\mathrm{CO}_{2}$ profiles for the $« 5 \%$ flaming zone» case. Obviously in this case the algorithm is capable of determining the concentration profiles with high accuracy.

\section{Conclusions}

In order to improve the knowledge of the effect of emissions by wild fires and volcanic activities on the ecosystem it is necessary to quantify the amount of emitted gases; especially greenhouse gases. The FASA system presented in this paper aims at contributing to this task. It consists of the Fourier Transform spectrometer MIROR and the imaging system ABAS. Both are operated in parallel on board a research aircraft. ABAS provides images of high spatial resolution. These are used to provide additional information which is necessary to retrieve temperature and gas concentration profiles from the MIROR spectra. Results of a first calculation to demonstrate the feasibility of the retrieval algorithm were provided and show encouraging results. Of course, further investigations are nec- essary to describe the complete data evaluation chain. The aim of the FASA development is to provide parameters such as gas column or profile information of e.g., $\mathrm{CO}_{2}, \mathrm{H}_{2} \mathrm{O}, \mathrm{CO}$, and $\mathrm{SO}_{2}$, temperatures, and also combustion efficiency, and aerosol parameters. As a first application of FASA a measurement campaign over Mt. Etna, Italy is planned in 2003. It can be expected that in the future the FASA capabilities will also permit improved evaluation of satellite data.

\section{REFERENCES}

Haschberger, P. (1992): Entwicklung eines Fourierspektrometers mit rotierendem Retroreflektor, Ph.D. Thesis (Technical University Munich).

Haschberger, P. and E. LindermeIR (1996): Spectrometric inflight measurement of aircraft exhaust emissions, $J$. Geophys. Res., 101, 25995-26006

Lindermeir, E., S. Clausen, R.M. Geatches and E. Vivo (2001): Profiling Spectrometery to simultaneously investigate the spatial distribution of temperature and chemical species in aircraft exhausts (AEROPROFILE), Synthesis Rep. (European Commission, Project No. BE97-4467, Contract No. BRPR-CT-97-0509).

Page, S.E., F. Siegert, J.O. Rieley, H-D.V. Boehm, A. Jaya 
and S. LIMIN (2002): The amount of carbon released from peat and forest fires in Indonesia during 1997, Nature, 420, 61-65.

PereirA, A.C. and A.W. Setzer JR. (1996): Comparison of fire detection in savannas using AVHRR's channel 3 and TM images, Int. J. Remote Sensing, 17 (10), 1927-1937.

SCHIMPF, B.A. (1999): Analysis of far infrared spectra for the determination of the middle atmospheric $\mathrm{OH}$ concentration, Ph.D. Thesis (University of Bremen).
Stocks, B.J., D.R. CAHoon, J.S. Levine, W.R. Cofer and T.J. LYNHAM (1996): Major 1992 fires in Central and Eastern Siberia: satellite and fire danger measurements, in Fire in Ecosystems of Boreal Eurasia, edited by J.G. GoldAMmER and V.V. FurYaEV (Kluwer Academic Publ., Dordrecht, The Netherlands), 139-150.

Worden, H., R. BEER and C.P. RinSLAND (1997): Airborne infrared spectroscopy of 1994 western wildfires, $J$. Geophys. Res., 102, 1287-1300. 